\title{
Do all cases of vasa previa need cesarean delivery? a case report and review of the literatures
}

\begin{abstract}
We herein describe our experiences in vaginal deliveries for women with prenatally diagnosed vasa previa (VP).

Case 1: A 29-year-old uniparous woman was referred to us at $34^{+4}$ gestational weeks. Trans vaginal ultrasonography (TV-USG) showed a membranous fetal vein located $1.1 \mathrm{~cm}$ apart from the internal cervical os. At $34^{+6}$ gestational weeks, spontaneous rupture of membranes (ROM) occurred, and labor was naturally initiated at $36^{+1}$ gestational weeks. The progression of labor was favorable and a non-anemic male baby weighing $2290 \mathrm{~g}$ was vaginally delivered 9.5 hours after the onset of labor.
\end{abstract}

Case 2: A 32-year-old primiparous woman was referred at $39^{+2}$ gestational weeks. TV-USG showed a pair of membranous fetal vein and artery located $1.0 \mathrm{~cm}$ apart from the internal cervical os. At $40^{+4}$ gestational weeks, spontaneous ROM occurred and labor was naturally initiated 2 hours later. After labor augmentation with intravenous prostaglandin $\mathrm{F} 2 \alpha$, a non-anemic female baby weighing $2715 \mathrm{~g}$ was vaginally delivered 22 hours after the onset of labor.

These experiences and a review of past case reports suggest that an early minimallytraumatic amniotomy, avoidance of excessive cervical manipulation, and the shortening of the expulsive stage could contribute to the prevention of VP rupture.

Keywords: amniotomy, color doppler, fetal exsanguinations, rupture of membranes, vaginal delivery, variable deceleration
Volume 2 Issue 5 - 2017

\author{
Atsuko Taga, Yukiyasu Sato Chieko Sakae, \\ Yumiko Satake, Ikuko Emoto, Shunsuke \\ Maruyama, Tomoko Kim \\ Department of Obstetrics and Gynecology, Otsu Red Cross \\ Hospital, Japan
}

Correspondence: Yukiyasu Sato, Department of Obstetrics and Gynecology, Otsu Red Cross Hospital

| I-35 Nagara, Otsu 520-85 I I, Shiga, Japan, Tel 81-77-522-4|3|, Fax 81-77-525-8018, Email yukiyasu@kuhp.kyoto-u.ac.jp

Received: May 06, 2017 | Published: August 21, 2017

\section{Introduction}

Vasa previa (VP) is defined as fetal vessels unsupported by either placenta or umbilical cord coursing within the membranes in close proximity to the internal cervical os. ${ }^{1}$ The membranous fetal vessels can either lead from the placenta to a velamentous cord insertion (type 1) or connect the main bulk of the placenta to a succenturiate lobe (type 2). ${ }^{2}$ The localization of the membranous fetal vessels can be classified as overlying (located directly over the internal os) or low-lying (located apart from the internal os but in front of the fetal presenting part). ${ }^{3}$

It is possible for membranous fetal vessels to tear at membrane rupture, or during cervical dilation, and cause fetal exsanguinations. ${ }^{4}$ In the multicenter study consisting of 155 pregnancies complicated with VP, Oyelese et al. ${ }^{5}$ demonstrated that perinatal mortality rate in VP cases diagnosed prenatally was significantly lower than in those not diagnosed prenatally ( 2 perinatal deaths/ 61 cases vs. 53 perinatal deaths/94 cases). Since elective cesarean sections were performed in $69 \%$ of the cases diagnosed prenatally, and only $4 \%$ in those not diagnosed prenatally, the authors concluded that women with VP should be electively delivered by cesarean section at 35 gestational weeks before the onset of labor or the rupture of membranes. ${ }^{5}$

Although the Oyelese's results are convincing enough to draw the above conclusion, inherent selection bias should be taken into account. VP cases that were not prenatally diagnosed and had an uneventful vaginal delivery would not come to be noticed and thus were unintentionally excluded from the study. Hasegawa et al. ${ }^{6}$ reported that systematic first-trimester ultrasound detected $10 \mathrm{VP}$ cases out of 3647 cases, calculating the incidence of $1 / 365 .^{6}$ This figure is far higher than the widely accepted incidence of $1 / 2500$ estimated from several case series, ${ }^{7}$ suggesting that the majority of VP cases have been over looked. Therefore, while safe vaginal delivery is clearly out of the question in the presence of overlying VP, it remains to be determined whether all cases with low-lying VP require elective cesarean section. ${ }^{2}$

Here, we describe our experience in two cases of prenatally diagnosed low-lying VP where vaginal deliveries were attempted with extreme caution. We also review past case reports that described details of the clinical course leading to the VP rupture to find out certain preventive measures against VP rupture during vaginal delivery.

\section{Case report}

\section{Case I}

A 29-year-old, para 1, gravida1 (1 normal vaginal delivery) woman was referred to our hospital due to the suspicion of V Pat $34^{+4}$ gestational weeks. A low-lying placenta that had been noted at 24 gestational weeks was spontaneously resolved as pregnancy progressed, visualizing the umbilical vessel running near the internal os. Color Doppler trans vaginal ultrasonography (TV-USG) confirmed a membranous fetal vein located $1.1 \mathrm{~cm}$ apart from the internal cervical os with a cervical length of $3.3 \mathrm{~cm}$ (Figure 1A). Transabdominal ultrasonography (TAUSG) showed a normally grown fetus in cephalic presentation and the placenta attached to the posterior uterine wall. The running course of the fetal vessels inferred from the TA-USG and TV-USG findings is illustrated in Figure 1B. The patient was admitted to the hospital under the diagnosis of low-lying VP type 1. After being fully informed 
of the possible risk for fetal exsanguination from VP rupture, the patient consented to a vaginal delivery with preparation for immediate cesarean section. At $34^{+6}$ weeks of gestation, spontaneous rupture of membranes (ROM) occurred. Although the location of VP remained unchanged in TV-USG, no abnormal uterine bleeding was noted, and fetal heart rate tracing showed a reassuring pattern. Prophylactic intravenous administration of ampicillin ( $2 \mathrm{~g} /$ day) was instituted and complete blood cell count and CRP was monitored every day to detect the possible sign of intrauterine infection. Intravenous administration of erythromycin ( $1 \mathrm{~g} /$ day) was added from $35^{+2}$ gestational weeks due to mild elevation of CRP $(1.2 \mathrm{mg} / \mathrm{dl})$, and CRP stayed negative after $35^{+4}$ gestational weeks. Fetal well-being was confirmed by daily fetal heart rate tracing, and the uterine cervical status was checked every day mainly by TV-USG, rather than by internal examination, in order to avoid the accidental injury of VP. No cervical shortening was noted, and the distance between the internal os and VP remained unchanged. At $36^{+1}$ gestational weeks, labor was spontaneously initiated. In TV-USG performed 2hours after the labor onset, cervical length had shortened to $1.3 \mathrm{~cm}$, and VP had moved upwards outside the observable area. The progression of labor was favorable and 9.5hours after the labor onset, and a male baby weighing $2290 \mathrm{~g}$ was vaginally delivered with Apgar scores of 8 at 1minute and 9 at 5 minutes. Umbilical arterial blood $\mathrm{pH}$ was 7.235 , and the neonate was not anemic. The partogram is shown in Figure 1C. Continuous fetal heart tracing showed that repetitive variable deceleration coincident with every uterine contraction (Figure 1D) persisted for 0.5 hours throughout the expulsive stage, and the placenta proved to have velamentous insertion of the umbilical cord without ruptured vessels (Figure 1E). The postpartum courses of mother and baby were both uneventful and both were discharged from the hospital on the 5th day.

\section{Figure 1}
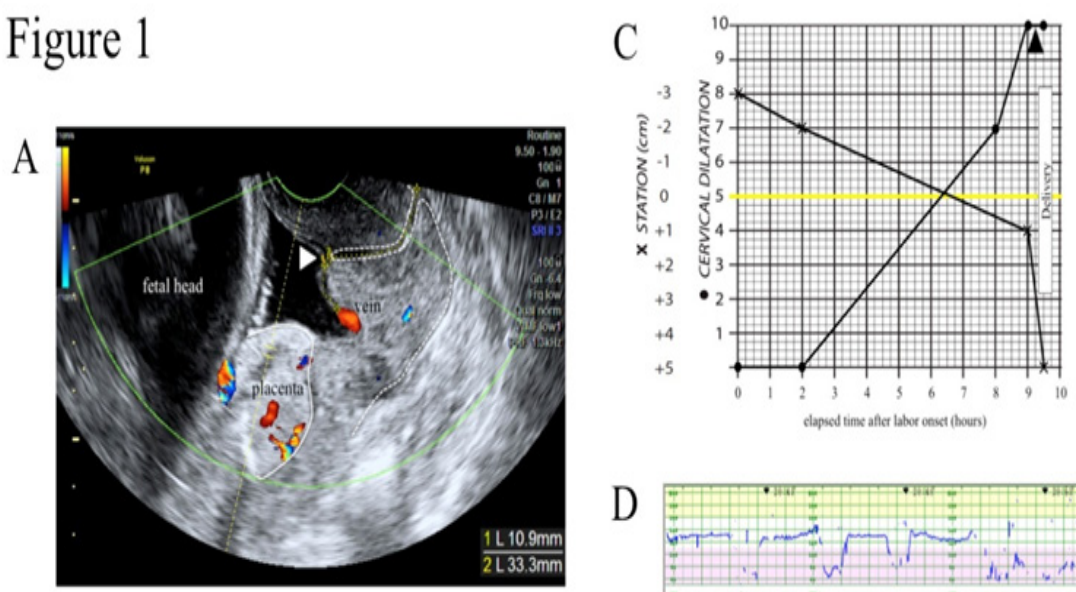

D
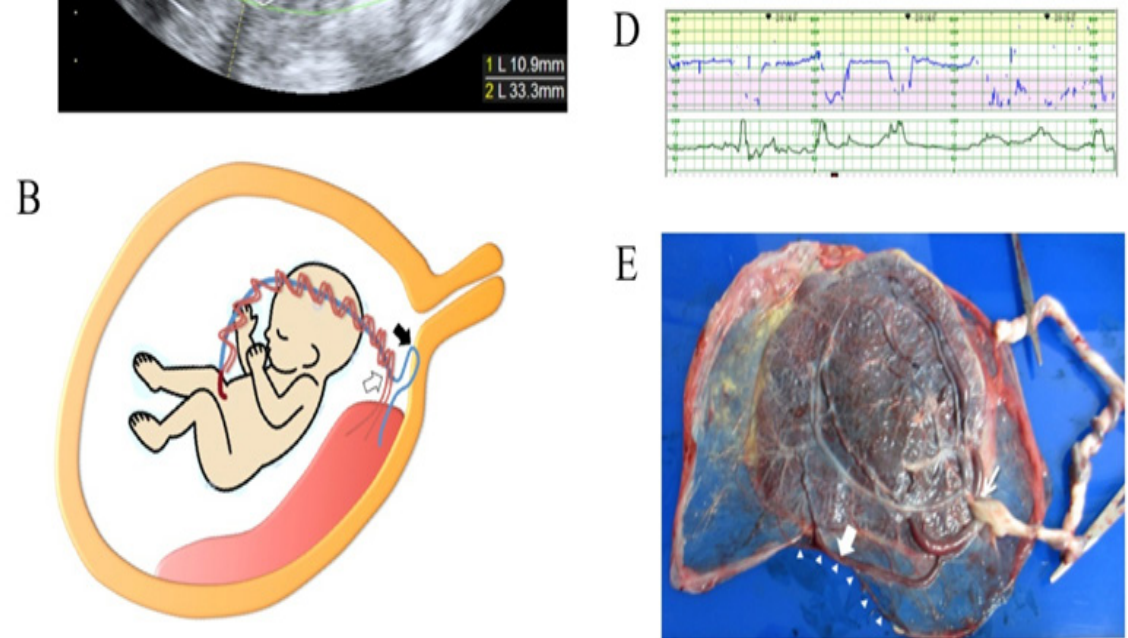

E

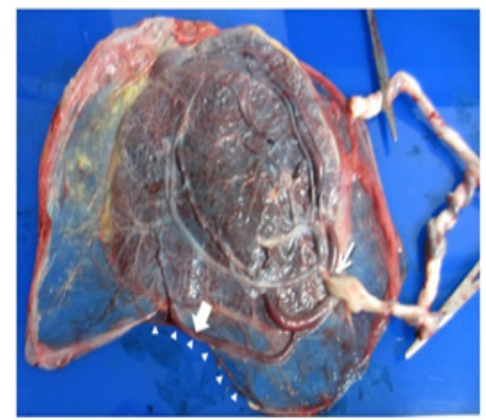

Figure I Clinical presentations of case I.

(A) Color Doppler transvaginal ultrasonogram at $34^{+4}$ gestational weeks, showing a membranous fetal vein located $\mathrm{I} . \mathrm{I} \mathrm{cm}$ apart from the internal cervical os with cervical length of $3.3 \mathrm{~cm}$. Open arrowhead indicates the internal cervical os.

(B) Illustration showing the running course of fetal vessels that was inferred from the transabdominal and transvaginal ultrasonographic findings. Open arrow indicates the velamentous insertion site of the umbilical cord. Filled arrow indicates the segment of fetal vein visualized in (A).

(C) Partogram plotting the station and cervical dilation from the labor onset until the delivery.

(D) Cardiotocogram at the time period indicated by filled arrowhead in (C). Fetal heart rate tracing shows repetitive variable deceleration coincident with every uterine contraction. This fetal heart rate pattern persisted for 0.5 hours throughout the expulsive stage.

(E) Macroscopic findings of delivered placenta and fetal membranes in case I, showing velamentous insertion of the umbilical cord (thin arrow). Note a membranous fetal vein (thick arrow) running adjacent to the site of membrane rupture (dotted line). 


\section{Case 2}

A 32-year-old, para 1, gravida 0 (1 spontaneous abortion) woman was referred to our hospital due to the suspicion of VP at $39^{+2}$ gestational weeks. Color Doppler TV-USG confirmed a pair of membranous fetal vein and artery located $1.0 \mathrm{~cm}$ apart from the internal cervical os with a cervical length of $2.0 \mathrm{~cm}$ (Figure 2A). TA-USG showed a normally grown fetus in cephalic presentation and the placenta attached to anterior uterine wall. The running course of the fetal vessels inferred from the TA-USG and TV-USG findings is illustrated in Figure 2B. The patient was admitted to the hospital under the diagnosis of lowlying VP type 1. After being fully informed of the possible risk for fetal exsanguinations from VP rupture, the patient consented to a vaginal delivery with preparation for immediate cesarean section. Since oral dinoprostone (prostaglandin E2 $\alpha$ ) for two consecutive days (6tablets/day) and intravenous dinoprost (prostaglandin F2 $\alpha$ ) for one day (up to $25 \mathrm{~g} /$ minute) failed to induce effective labor pains, we decided to wait for spontaneous onset of labor. At $40^{+4}$ gestational weeks, spontaneous ROM occurred, and labor was naturally initiated 2hours later. In TV-USG performed 14hours after the labor onset, cervical length had shortened to $1.0 \mathrm{~cm}$ and VP had moved upwards outside the observable area. At $40^{+5}$ gestational weeks, intravenous dinoprost (prostaglandin F2) was instituted 15hours after the labor onset to augment uterine contractions. Afterwards, the progression of labor was favorable, and 22 hours after the labor onset, a female baby weighing $2715 \mathrm{~g}$ was vaginally delivered with Apgar scores of 8 at 1 minute and 8 at 5minutes. Umbilical arterial blood $\mathrm{pH}$ was 7.154 and the neonate was not anemic. The partogram is shown in Figure 2C. Continuous fetal heart tracing showed that repetitive variable deceleration coincident with every uterine contraction (Figure 2D) persisted for 3hours throughout the expulsive stage. The placenta proved to have velamentous insertion of the umbilical cord without ruptured vessels (Figure 2E). The postpartum courses of mother and baby were both uneventful and both were discharged from the hospital on the 5th day.

\section{Figure 2}
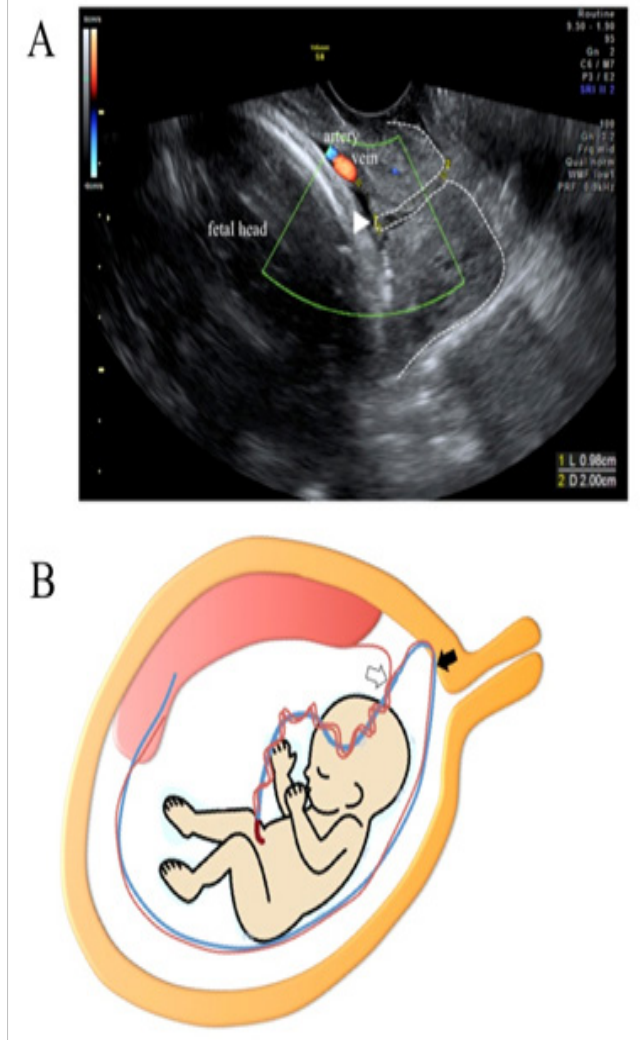

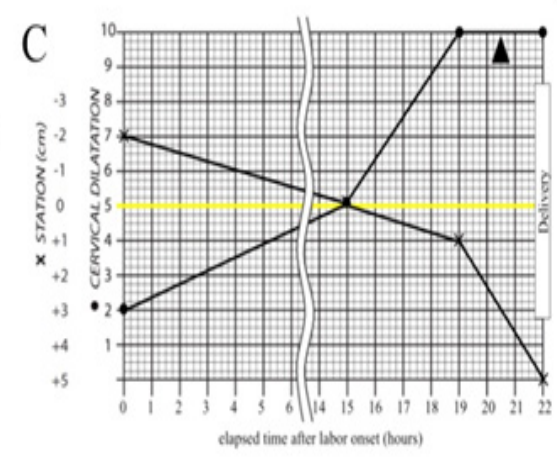

D

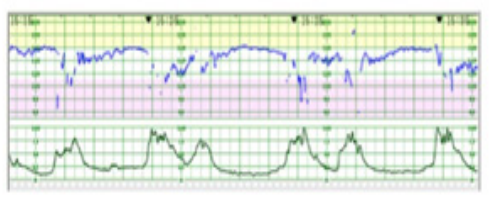

E

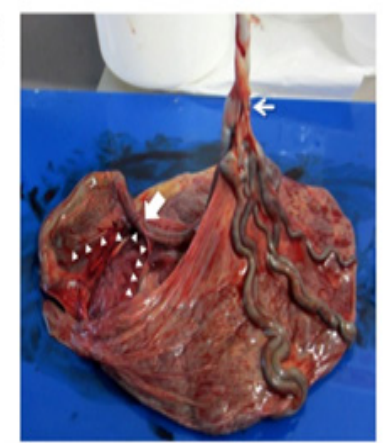

Figure 2 Clinical presentations of case 2.

(A) Color Doppler transvaginal ultrasonogram at 39+2 gestational weeks, showing a pair of membranous fetal vein and artery located I.0 $\mathrm{cm}$ apart from the internal cervical os with the cervical length of $2.0 \mathrm{~cm}$. Open arrowhead indicates the internal cervical os.

(B) Illustration showing the running course of fetal vessels that was inferred from the transabdominal and trans vaginal ultra sonographic findings. Open arrow indicates the velamentous insertion site of the umbilical cord. Filled arrow indicates the segment of fetal vessels visualized in (A).

(C) Partogram plotting the station and cervical dilation from the labor onset until the delivery.

(D) Cardiotocogram at the time period indicated by filled arrowhead in (C). Fetal heart rate tracing shows repetitive variable deceleration coincident with every uterine contraction. This fetal heart rate pattern persisted for 3hours throughout the expulsive stage.

(E) Macroscopic findings of delivered placenta and fetal membranes in case 2, showing velamentous insertion of the umbilical cord (thin arrow). Note a pair of membranous fetal vein and artery (thick arrow) running adjacent to the site of membrane rupture (dotted line). 
Table I Clinical courses of 17 cases suffering from rupture of vasa previa

\begin{tabular}{|c|c|c|c|c|c|c|c|c|}
\hline \multirow{2}{*}{ Case no. } & \multirow{2}{*}{ Year } & \multirow{2}{*}{$\begin{array}{l}\text { Gestational } \\
\text { weeks }\end{array}$} & \multicolumn{3}{|c|}{ Situation at rupture of vasa previa } & \multirow{2}{*}{$\begin{array}{l}\text { Delivery } \\
\text { mode }\end{array}$} & \multirow{2}{*}{$\begin{array}{l}\text { Live birth / } \\
\text { stillbirth }\end{array}$} & \multirow{2}{*}{ Ref. } \\
\hline & & & Labor pain & $\begin{array}{l}\text { Cervical } \\
\text { dilation }\end{array}$ & Rupture of membranes & & & \\
\hline I & 1984 & 39 & $(+)$ & $4 \mathrm{~cm}$ & spontaneous & eCS & live birth & 9 \\
\hline 2 & 1987 & 40 & $(+)$ & $2 \mathrm{~cm}$ & spontaneous & eCS & live birth & 10 \\
\hline 3 & 1987 & 40 & $(+)$ & $6-7 \mathrm{~cm}$ & spontaneous & VD & stillbirth & 10 \\
\hline 4 & 1990 & 40 & $(+)$ & $5 \mathrm{~cm}$ & artificial & eCS & live birth & 12 \\
\hline 5 & 1995 & 36 & $(+)$ & $8 \mathrm{~cm}$ & artificial & VD & live birth & 13 \\
\hline 6 & 1995 & 39 & $(+)$ & $3-4 \mathrm{~cm}$ & artificial & eCS & live birth & 13 \\
\hline 7 & 1987 & 39 & $(-)$ & $2 \mathrm{~cm}$ & spontaneous & $\mathrm{eCS}$ & stillbirth & 11 \\
\hline 8 & 2003 & 38 & $(-)$ & closed & spontaneous & eCS & stillbirth & 14 \\
\hline 9 & 2010 & 36 & $(-)$ & $3 \mathrm{~cm}$ & artificial & VD & live birth & 15 \\
\hline 10 & 1982 & 35 & $(+)$ & $6 \mathrm{~cm}$ & $(-)$ & VD & stillbirth & 16 \\
\hline II & 1985 & 38 & $(+)$ & $3 \mathrm{~cm}$ & artificial (I.5h before) & eCS & live birth & 17 \\
\hline 12 & 1988 & 39 & $(+)$ & $3 \mathrm{~cm}$ & $(-)$ & eCS & live birth & 18 \\
\hline 13 & 1988 & 38 & $(+)$ & $\mathrm{Icm}$ & $\begin{array}{l}\text { spontaneous ( } 0.5 \mathrm{~h} \\
\text { before) }\end{array}$ & eCS & live birth & 19 \\
\hline 14 & 1990 & 39 & $(-)$ & $\mathrm{Icm}$ & $(-)$ & eCS & live birth & 20 \\
\hline 15 & 1990 & 34 & $(+)$ & $\begin{array}{l}10 \mathrm{~cm} \\
\text { (expulsive } \\
\text { stage) }\end{array}$ & $\begin{array}{l}\text { spontaneous ( } 5 \mathrm{~h} \\
\text { before) }\end{array}$ & VD & live birth & 21 \\
\hline 16 & 2012 & 37 & $(+)$ & $\begin{array}{l}10 \mathrm{~cm} \\
\text { (expulsive } \\
\text { stage) }\end{array}$ & $\begin{array}{l}\text { spontaneous (? h } \\
\text { before) }\end{array}$ & VD & live birth & 22 \\
\hline 17 & 1991 & 33 & $(-)$ & $\begin{array}{l}\text { closed } \\
\text { (cerclage) }\end{array}$ & $\begin{array}{l}\text { spontaneous ( } 3 \mathrm{~h} \\
\text { before) }\end{array}$ & eCS & live birth & 23 \\
\hline
\end{tabular}

eCS, emergency cesarean section; VD, vaginal delivery, Rupture of vasa previa occurred at rupture of membranes in cases $1-9$, during cervical dilation in cases $10-14$, during the expulsive stage of labor in case 15 and 16 , and after cervical cerclage in case 17

\section{Discussion}

Owing to the recent advance and prevalence of color Doppler ultrasonography, the number of VP cases diagnosed prenatally has increased. Nevertheless, due to Oyelese's recommendation that women with VP should be electively delivered by cesarean section at 35 gestational weeks, ${ }^{5}$ a trial of vaginal delivery after the diagnosis of VP has never before appeared in English literatures. Accordingly, this is the first report of two such cases.

A precise definition for low-lying VPvis-à-vis their distance between the membranous fetal vessels and the internal cervical os has yet to be determined. In placenta previa, it was demonstrated that the risk of peripartum bleeding becomes higher when the placental edge is located within $2 \mathrm{~cm}$ of the internal os. ${ }^{8}$ Extrapolating from this, Rebarber et al. ${ }^{1}$ proposed the same cut-off value of $2 \mathrm{~cm}$ in the definition of low-lying VP, although there has been no convincing evidence that perinatal mortality becomes higher when the membranous fetal vessels are located within $2 \mathrm{~cm}$ of the internal os. ${ }^{1}$ Moreover, analogous with cases of placenta previa, the upward migration and resolution of a prenatally diagnosed VP throughout the gestation has been documented. ${ }^{1}$ Thus, the definitive diagnosis of VP should be made in the third trimester. In our two cases, the distances between the membranous fetal vessel and the internal os was $1.1 \mathrm{~cm}$ at $34^{+4}$ gestational weeks in case 1 and $1.0 \mathrm{~cm}$ at $39^{+2}$ gestational weeks in case 2 , leading to the diagnoses of low-lying VP.

To find out certain preventive measures against VP ruptured using vaginal delivery, we reviewed only case reports that described details of the clinical course leading to the VP rupture. Since 1980, 17 of such cases could be identified (Table 1). In all of these cases, VP was not diagnosed prenatally and, therefore, a determination of overlying or low-lying type was not made. VP was ruptured at spontaneous or artificial ROM in 9 case, ${ }^{9-15}$ during cervical dilation in 5 cases, ${ }^{16-20}$ and during the expulsive stage of labor in 2 cases. ${ }^{21,22}$ In the one remaining case, VP was ruptured after cervical cerclage was performed due to preterm $\mathrm{ROM},{ }^{23}$ suggesting that excessive cervical manipulation could induce the rupture of VP. Among the 17 cases, labor pains accompanied the rupture of VP in 12cases. In these 12 cases, high intra-amniotic pressure at the time of ROM, cervical dilation, or bearing down could be one of the reasons behind the VP rupture. In this respect, early minimally-traumatic amniotomy (e.g. using fine needle) as soon as the membrane becomes accessible by vaginal inspection could relieve the increase of intra-aminiotic pressure during uterine contractions and, thus, could also reduce the risk of VP rupture. As might be expected, such amniotomy should be 
performed with extreme caution after confirming the location of VP by color Doppler TV-USG. Fortunately, spontaneous ROM without VP rupture preceded the onset of labor in both of our cases.

It is widely recognized that the upper uterine cervix is transformed and incorporated into the lower uterine segment as labor progresses. Accordingly, low-lying VP is expected to migrate upwards during labor. In both of our cases, although the location of VP remained unchanged after the spontaneous ROM, VP migrated upwards after the onset of labor. This upward migration could contribute to the accomplishment of the vaginal delivery without VP rupture.

Typically, a sinusoidal fetal heart rate pattern follows fetal exsanguination from the ruptured VP. ${ }^{16,19,20,23}$ In both of our cases, a sinusoidal heart rate pattern was not observed. Instead, a repetitive variable deceleration, which was considered to result from the compression of VP by the fetal presenting part, persisted throughout the expulsive stage. Such compression could herald the rupture of VP. In fact, VP rupture that developed during the expulsive stage has been reported..$^{21,22}$ Thus, we would recommend that the expulsive stage should be shortened using vacuum extraction or forceps.

\section{Conclusion}

We experienced vaginal deliveries in two cases of low-lying VP that had been prenatally diagnosed. These experiences and review of past case reports suggest that an early minimally-traumatic amniotomy, avoidance of excessive cervical manipulation, and the shortening of the expulsive stage could contribute to the prevention of VP rupture. Although we might have been only lucky due to the fact that spontaneous ROM without VP rupture preceded the onset of labor, we believe that vaginal delivery can be safely accomplished in certain cases of low-lying VP. More experience is necessary to identify the characteristics of VP that allows the trial of vaginal delivery.

\section{Acknowledgements}

None.

\section{Conflict of interest}

The authors report no conflicts of interest. The authors alone are responsible for the content and writing of the paper.

\section{References}

1. Rebarber A, Dolin C, Fox NS, et al. Natural history of vasa previa across gestation using a screening protocol. J Ultrasound Med. 2014;33(1):141-147.

2. Catanzarite V, Maida C, Thomas W, et al. Prenatal sonographic diagnosis of vasa previa: ultrasound findings and obstetric outcome in ten cases. Ultrasound Obstet Gynecol. 2001;18(2):109-115.

3. Bronsteen R, Whitten A, Balasubramanian M, et al. Vasa previa: clinical presentations, outcomes, and implications for management. Obstet Gynecol. 2013;122(2 Pt 1):352-357.
4. Fung TY, Lau TK. Poor perinatal outcome associated with vasa previa: is it preventable? A report of three cases and review of the literature. Ultrasound Obstet Gynecol. 1998;12(6):430-433.

5. Oyelese Y, Catanzarite V, Prefumo F, et al. Vasa previa: the impact of prenatal diagnosis on outcomes. Obstet Gynecol. 2004;103(5 Pt 1):937-942.

6. Hasegawa J, Nakamura M, Ichizuka K, et al. Vasa previa is not infrequent. J Matern Fetal Neonatal Med. 2012;25(12):2795-2796.

7. Oyelese KO, Turner M, Lees C, et al. Vasa previa: an avoidable obstetric tragedy. Obstet Gynecol Surv. 1991;54(2):138-145.

8. Matsubara S, Ohkuchi A, Kikkawa M, et al. Blood loss in low-lying placenta: placental edge to cervical internal os distance of less vs. more than $2 \mathrm{~cm} . J$ Perinat Med. 2008;36(6):507-512.

9. Silva PD, Stoskopf CG, Keegan KA, et al. Use of fetal scalp hematocrit in the diagnosis of severe hemorrhage from vasa previa. Am J Obstet Gynecol. 1985;153(3):307-308.

10. Dougall A, Baird $\mathrm{CH}$. Vasa praevia--report of three cases and review of literature. Br J Obstet Gynaecol. 1987;94(7):712-715.

11. Jones KP, Wheater AW, Musgrave W. Simple test for bleeding from vasa praevia. Lancet. 1987;2(8573):1430-1431.

12. Bhattacharyya N. Vasa praevia.JIndian MedAssoc. 1990;88(8):229-230.

13. Schellpfeffer MA. Improved neonatal outcome of vasa previa with aggressive intrapartum management. A report of two cases. J Reprod Med. 1995;40(4):327-332.

14. Robert JA, Sepulveda W. Fetal exsanguination from ruptured vasa previa: still a catastrophic event in modern obstetrics. J Obstet Gynaecol. 2003;23(5):574

15. Papathanasiou D, Witlox R, Oepkes D, et al. Monochorionic twins with ruptured vasa previa: double trouble! Fetal Diagn Ther. 2010;28(1):48-50.

16. Antoine C, Young BK, Silverman F, et al. Sinusoidal fetal heart rate pattern with vasa previa in twin pregnancy. $J$ Reprod Med. 1982;27(5):295-300.

17. Hanretty KP. Ruptured vasa praevia. Scott Med J 1985;30(2):115-116.

18. Reuter KL, Davidoff A, Hunter T. Vasa previa. J Clin Ultrasound. 1988;16(5):346-348.

19. Tollison SB, Huang PH. Vasa previa. A case report. J Reprod Med. 1988;33(3):329-330.

20. Gantt PA, Bird JS, Randall GW. Sinusoidal fetal heart rate pattern with vasa previa. J Tenn Med Assoc. 1990;83(8):393-394.

21. Good MC, Copas PR, Kleinman GE, et al. Vasa previa. J Tenn Med Assoc. 1990;83(10):499-501.

22. Carnide $\mathrm{C}$, Jeronimo $\mathrm{M}$, Faria $\mathrm{D}$, et al. Twin pregnancy complicated by vasa previa. BMJ Case Rep. 2012.

23. Kruitwagen RF, Nijhuis JG. Ruptured vasa praevia indicated by a sinusoidal fetal heart rate pattern: a case report. Eur J Obstet Gynecol Reprod Biol. 1991;39(2):147-150. 